Wenwen Lin

Yuejun Zhang

https://doi.org/10.21278/TOF.44107

ISSN 1333-1124

eISSN 1849-1391

\title{
INTEGRATED PRODUCTION AND MAINTENANCE PLANNING FOR FLOW LINE SYSTEMS
}

\begin{abstract}
Summary
This study extends the investigation of the capacitated lot-sizing problem to the production and maintenance planning in unreliable flow line systems. An integrated modelling framework is proposed with the aim of seeking a cost-optimal plan for both production and maintenance. In the model, preventive maintenance is scheduled to avoid unplanned failures, and corrective maintenance is carried out in any machine in which an unplanned failure occurs. A regression-based approximation approach was introduced to calculate the production time under random failures. Then, the integrated planning model can be solved by any commercial optimization software. The numerical example demonstrates that the integrated model guarantees the effectiveness of the production and maintenance plan. It also showed that the buffer capacity has significant effects on the capacity utilization.
\end{abstract}

Key words: $\quad$ production planning; maintenance; flow line system; simulation; polynomial regression

\section{Introduction}

It is natural that a machine deteriorates and then fails during production. Any machine failure might lead to the whole production line breaking down. Therefore, maintenance is necessary to restore machines. In the past several decades, many maintenance and replacement models have been proposed from the perspective of cost, availability or reliability $[1,2]$.

Integrating maintenance into the production planning has been highlighted already in the paper by Weinstein and Chung [3]. Gu et al. [4] searched for hidden maintenance opportunities to perform maintenance tasks without affecting the system throughput. Arab [5] investigated a maintenance scheduling problem which incorporates the real-time information from workstations and work-in-process inventories.

\subsection{Integrated planning of production and maintenance}

Due to stoppages caused by machine failures and maintenance, the efficiency decreases over the planning horizon. When machine failures occur, corrective maintenance (CM) is carried out to replace the worn-out component and keeps machines operational. Preventive 
maintenance (PM), on the other hand, is scheduled in advance to avoid unplanned failures and it reduces the cost caused by stoppages.

Weinstein and Chung [3] proposed an aggregated production planning (APP) model with maintenance operations at multiple levels and motivated a considerable number of follow-up papers listed in Table 1. Mark " $\sqrt{ }$ " means that corresponding maintenance is used while "-" means that such maintenance is not used. Most of the problems considered in the mentioned papers can be described as a special case of the capacitated lot sizing problem (CLSP), which is a classical production planning model that contains the essential requirements for the planning of the batch production. These CLSP models are conceived as single-stage systems in terms of the system configuration. Further, Ramezanian et al. [6] proposed an integrated planning model for a multi-level production system. However, the machine capacity was tackled separately.

Table 1 Literature about integrated planning of production and maintenance

\begin{tabular}{|c|c|c|c|c|c|}
\hline Literatures & Year & Model & PM & CM & System configuration \\
\hline Weinstein and Chung [3] & 1999 & APP & $\sqrt{ }$ & $\sqrt{ }$ & Parallel machines \\
\hline Aghezzaf et al. [7] & 2007 & CLSP & $\sqrt{ }$ & $\sqrt{ }$ & Single machine \\
\hline Aghezzaf and Najid [8] & 2008 & CLSP & $\sqrt{ }$ & $\sqrt{ }$ & Parallel machines \\
\hline Nourelfath et al. [9] & 2010 & CLSP & $\sqrt{ }$ & $\sqrt{ }$ & Multi-state system \\
\hline Najid et al. [10] & 2011 & CLSP & $\sqrt{ }$ & $\sqrt{ }$ & Single machine \\
\hline Nourelfath and Châtelet [11] & 2012 & CLSP & $\sqrt{ }$ & $\sqrt{ }$ & Multi-state machine \\
\hline Fitouhi and Nourelfath [12] & 2012 & CLSP & $\sqrt{ }$ & $\sqrt{ }$ & Single machine \\
\hline Alaoui-Selsouli et al. [13] & 2012 & CLSP & $\sqrt{ }$ & $\sqrt{ }$ & Single machine \\
\hline Machani and Nourelfath [14] & 2012 & CLSP & $\sqrt{ }$ & $\sqrt{ }$ & Multi-state system \\
\hline Lu et al. [15] & 2013 & CLSP & $\sqrt{ }$ & - & Single machine \\
\hline Wang [16] & 2013 & CLSP & $\sqrt{ }$ & $\sqrt{ }$ & Single machine \\
\hline Yalaoui et al. [17] & 2014 & CLSP & $\sqrt{ }$ & $\sqrt{ }$ & Parallel machines \\
\hline Fitouhi and Nourelfath [18] & 2014 & CLSP & $\sqrt{ }$ & $\sqrt{ }$ & Multi-state system \\
\hline Zhao et al. [19] & 2014 & CLSP & $\sqrt{ }$ & $\sqrt{ }$ & Single machine \\
\hline Fakher et al. [20] & 2015 & CLSP & $\sqrt{ }$ & $\sqrt{ }$ & Parallel machines \\
\hline Fakher et al. [21] & 2015 & CLSP & $\sqrt{ }$ & $\sqrt{ }$ & Parallel machines \\
\hline Aghezzaf et al. [22] & 2016 & CLSP & $\sqrt{ }$ & $\sqrt{ }$ & Single machine \\
\hline Beheshti-Fakher et al. [23] & 2016 & CLSP & $\sqrt{ }$ & $\sqrt{ }$ & Single machine \\
\hline Hnaien et al. [24] & 2016 & CLSP & $\sqrt{ }$ & $\sqrt{ }$ & Single machine \\
\hline Erfanian and Pirayesh [25] & 2016 & APP & $\sqrt{ }$ & $\sqrt{ }$ & Parallel machine \\
\hline Nourelfath et al. [26] & 2016 & CLSP & $\sqrt{ }$ & - & Single machine \\
\hline Shamsaei and Vyve [27] & 2016 & CLSP & $\sqrt{ }$ & - & Single machine \\
\hline Fakher et al. [28] & 2017 & CLSP & $\sqrt{ }$ & $\sqrt{ }$ & Parallel machines \\
\hline
\end{tabular}

Many papers deal with multi-stage systems without considering machine maintenance. Abdul-Kader [29] provided a planning model that incorporates the capacity constraints from the operational level. To estimate the capacity, machine failures were treated as fictive products, and then the model could be converted into a linear programming model. Diaz and Handl [30] considered a real-world production planning problem, where a hybrid optimization approach with simulation and genetic algorithm was used to support decision making. 


\subsection{Optimization approaches}

Simulation-based approaches take advantage over analytical approaches in the production and maintenance planning. They are applied by adjusting non-deterministic parameters iteratively according to the simulation results, until the target values of the performance indicators are reached. Caggiano et al. [31] combined the CLSP optimization and system simulation by implementing a feedback loop involving the capacity. Chu et al. [32] formulated a bi-level program, where the planning and the scheduling problems are solved iteratively via service level constraints. Ponsignon and Mönch [33] evaluated a planning algorithm for master planning while taking the execution level into account. The simulation-based optimization improves the feasibility of the production plan, but the computational time is never short due to the iteration of simulation.

Apart from the simulation-based approaches, some approximation approaches are developed to address the complexities as well. Mehrjoo and Bashiri [34] proposed a decision support tool for production planning based on the multivariate statistical methods, including the principal component analysis and logistic regression. Stricker et al. [35] considered regression models for predicting an expected reject rate based on the recent system status, thus enabling a robust planning with volatile reject rates. In order to get a robust production plan, Gyulai et al. [36] took the capacity requirements as a general function of the assembled products in the simulation. These papers showed that some approximation approaches could be used in the production planning of some cases instead of simulation-based approaches. What is more, the former approach is always more efficient than the latter.

\subsection{Outline}

In an unreliable flow line system, analytic approaches failed to calculate the production time for any given lot, while simulations are time-consuming. The contribution of this paper can be summarized as follows: (i) a mathematical model for CLSP-M with both PM and CM considered in an unreliable flow line system is proposed; (ii) a regression-based approximation approach for capacity constraints is introduced, and then the model can be solved by any commercial optimization software.

The remainder of this paper is structured as follows: Section 2 gives the problem description and the integrated modeling framework. Section 3 introduces the regression-based approximation approach and proves its accuracy. A numerical example is given in Section 4 to validate the effectiveness of this modelling approach. In Section 5, conclusions are drawn and new research directions are discussed.

\section{Problem description}

In the CLSP-M model, PM and CM are introduced and need to be coordinated with the production tasks. The aim is to generate a cost-efficient plan for both production and maintenance.

\subsection{Integrated planning of production and maintenance}

Consider a flow line consisting of $M$ machines and $M-1$ buffers, as shown in Fig. 1. The line produces a set of products over a finite planning horizon of $T$ periods. The flow line consists of failure-prone machines arranged in series. Machines are economically and structurally independent. 


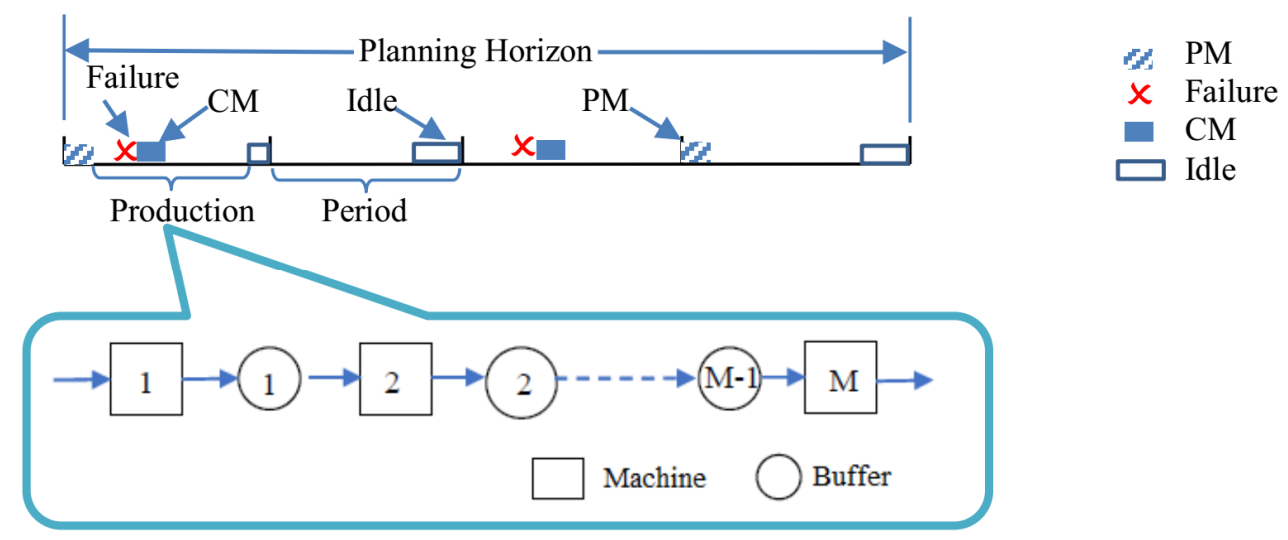

Fig. 1 Production and maintenance operations within a planning horizon

The following assumptions are made in this paper:

1) Each period has a deterministic demand for each kind of product to be satisfied.

2) All periods have the same length.

3) The set-up is carried out when switching from one kind of product to another.

4) There is no initial stock or backorder/shortage for any product.

5) The first machine is never starved and the last machine is never blocked.

6) At each stage, the finished products are released piece by piece and constitute the lot at the end.

7) The products in the buffer storages follow the first-in-first-out (FIFO) rule.

8) The processing time and the repair time for each machine are constant and predetermined.

9) The processing time of a product is a unit time.

10) Each machine deteriorates with an increasing failure rate, and the mean-time-tofailure (MTTF) follows a Weibull distribution.

11) Whenever a machine has a failure, $\mathrm{CM}$ is initiated and the machine is recovered to the initial state.

12) $\mathrm{PM}$ is scheduled at the beginning of some production period considering varying workload, and the production line is as good as new after PM.

13) The whole lot is completed on the flow line during the same period.

\subsection{Model formulation}

The integrated planning of production and maintenance in the flow line is modelled as a CLSP-M model. The following notations are used in the model.

\section{Constants}

$N$

$B$

\section{Indices}

$t$

$m$ number of products

a number which is greater than the ideal yield of products in a period when no failure occurs and no maintenance is scheduled

$$
\begin{aligned}
& \text { product index }(i=1,2, \cdots, N) \\
& \text { planning period index }(t=1,2, \cdots, T) \\
& \text { machine index }(m=1,2, \cdots, M)
\end{aligned}
$$




\section{Parameters}

$d_{i t}$

K

$h_{i t}$

$c_{i t}$

$s_{i t}$

$c_{s}^{p}$

$c_{m}^{c}$

$t_{s}^{p}$

$t_{m}^{c}$

\section{Variables}

$y_{i t}$

$I_{i t}$

$x_{i t}$

$u_{t}$

$w_{m t}$

$z_{t}$

$Q_{i t}$

\section{Function}

$r_{m}$ demand for product $i$ at the end of period $t$

length of the production period

holding cost per unit of $i$ at the end of period $t$

production cost per unit $i$ in period $t$

set-up cost of producing product $i$ in period $t$

PM cost of the production line

CM cost of machine $m$

PM duration for the production line

CM duration for machine $m$

The mathematical model is given as follows.

The objective function of the model is shown in Equation (1) :

$$
\sum_{i=1}^{N} \sum_{t=1}^{T}\left(s_{i t} y_{i t}+c_{i t} x_{i t}+h_{i t} I_{i t}\right)+\sum_{t=1}^{T} c_{s}^{p} u_{t}+\sum_{m=1}^{M} \sum_{t=1}^{T} c_{m}^{c} w_{m t}
$$

The model is subjected to Eqs (2)-(7):

$$
\begin{aligned}
& x_{i t}+I_{i, t-1}-I_{i t}=d_{i t}, \forall i \in\{1,2, \ldots, N\}, \forall t \in\{1,2, \ldots, T\} \\
& x_{i t} \leq B y_{i t}, \forall i \in\{1,2, \ldots, N\}, \forall t \in\{1,2, \ldots, T\} \\
& \sum_{i=1}^{N} Q_{i t}+u_{t} t_{s}^{p m} \leq K, \forall t \in\{1,2, \ldots, T\} \\
& z_{t}=\left(1-u_{t}\right) z_{t-1}+\sum_{i=1}^{N} x_{i t}, \forall t \in\{1,2, \ldots, T\}
\end{aligned}
$$




$$
\begin{aligned}
& w_{m t}=\int_{\left(1-u_{t}\right) z_{m, t-1}}^{z_{m t}} r(\tau)_{m} \mathrm{~d} \tau, \forall m \in\{1,2, \cdots, M\}, \forall t \in\{1,2, \ldots, T\} \\
& x_{i t}, I_{i t} \geq 0, y_{i t}, u_{t} \in\{0,1\}, \forall i \in\{1,2, \ldots, N\}, \forall t \in\{1,2, \ldots, T\}
\end{aligned}
$$

The objective function (1) is composed of system set-up cost, production cost, inventory holding cost, PM cost and CM cost. Constraint (2) ensures the material balance in the production periods for each product. Constraint (3) relates the production variables to the binary set-up variables. Constraint (4) is the capacity constraint, which indicates that the time for production and PM should not be longer than $K$. Equation (5) derives the effective machine age at the beginning of each period, while Equation (6) calculates the expected failure number. Constraint (7) relates to non-negativity and integrality constraints.

\subsection{Capacity constraint}

As we can see in constraint (4), the capacity constraint requires simulating the production time of a given production lot under random failures. The simulation procedure is shown in Fig. 2.

\section{Notations}

$j$

product index in $x$

$p_{j m}$

start time of product $j$ on machine $m$

$q_{j m}$

depart time of product $j$ on machine $m$

$B_{m}$ capacity of intermediate buffer after machine $m$

$\tau_{j m}$ age of machine $m$ after processing product $j$

$v_{j m}$

$=1$, if a failure occurs during the processing of product $j$ on machine $m$, otherwise, $v_{j m}=0$

Product $j$ is processed on machine $m$ after it departs machine $m-1$ and product $j-1$ departs machine $m$; that yields

$$
p_{j m}=\max \left\{q_{j, m-1}, q_{j-1, m}\right\}
$$

If machine $m$ is not blocked, $q_{j m}$ depends on $p_{j m}$, the processing time and the potential failure; otherwise, it is equal to the depart time of product $j-1-B_{m}$ on machine $m+1$ (Eq. (9)).

$$
q_{j m}=\max \left\{p_{j m}+1+v_{j m} t_{m}^{c}, q_{j-B_{m}-1, m+1}\right\}
$$

Failure occurrence can be determined by the randomly generated failure instant $\varphi_{m f}$ and the machine age $\tau_{j m}$.

$Q_{i t}$ is equal to the depart time of the last product in the lot on the last machine (Eq. (10)).

$$
Q_{i t}=q_{j m}, j=x, m=M
$$


Input: machine number, processing time; failure distribution, $\mathrm{CM}$ duration, production quantity, buffer size

Output: production time

Step 1: generate a two-dimensional array of $\varphi_{m f}$

Step 2: calculate $p_{j m}=\max \left\{q_{j, m-1}, q_{j-1, m}\right\}$

Step 3: if $\tau_{j-1, m}<\varphi_{m f}<\tau_{j m}$, assign 1 to $v_{j m}$ and $f+1$ to $f$; otherwise, assign 0 to $v_{j m}$

Step 4: calculate $q_{j m}$ using Eq. (9) and assign $\tau_{j-1, m}+a_{i m}$ to $\tau_{j m}$

Step 5: if $m<M$, assign $m+1$ to $m$, then go to Step 2; if $m==M$ and $j<x$, assign 1 to $m$ and $j+1$ to $j$, then go to Step 2; if $m==M$ and $j==x$, assign $q_{j m}$ to $Q\left(x_{i t}\right)$, output is generated.

End the procedure.

Fig. 2 Simulation procedure of the production time

\section{Regression-based approximation approach}

Instead of running multiple simulations during the optimization of the CLSP-M model, a "black box" approach for calculating the production time is introduced. The objective in the "black box" approach is to find a model which describes production time with regression analysis and then decouples the simulation procedure and the model optimization. For a single machine following a Weibull lifetime distribution $W\left[\beta_{m}, \eta_{m}\right]$, the expected production time $\Psi(\bullet)$ can be regressed as follows:

$$
\psi(x)=x+\left(\frac{x}{\eta_{m}}\right)^{\beta_{m}} c_{m}^{c}
$$

where $\Psi(x)$ is the expected production time, $x$ is the quantity of produced products, and $\beta_{m}$ is the polynomial degree.

In prior research, $\beta_{m}$ is usually assigned a small value (i.e. first-order polynomial). The reason is that the variation of $x_{i t}$ is always kept at a low level, which means that the value of $\beta_{m}$ has less impact on the regression result [37]. Thus, a lower polynomial degree is sufficient for regression.

Consider a production line; any failure causes variations in stages which are compensated by buffer storages. In that case, the polynomial degree and the coefficients are adjusted to improve the regression performance. A second-order polynomial is adapted for regression, as shown in Eq. (12).

$$
\psi(x)=a_{1} x+a_{2} x^{2}+\varepsilon
$$

In the proposed model, $Q_{i t}$ can be replaced by $\Psi\left(z_{t}\right)-\Psi\left(\left(1-u_{t}\right) z_{t-1}\right)$. Then, the constraint (4) can be re-represented as follows:

$$
\psi\left(z_{t}\right)-\psi\left(\left(1-u_{t}\right) z_{t-1}\right)+u_{t} t_{s}^{p m} \leq K_{t}
$$

Using Eq. (13) instead of Eq. (4), the model can be solved by commercial solvers or heuristics.

The performance of the polynomial regression is examined in different configurations. Suppose that there are 1, 000 products to be processed by an unreliable flow line system with 10 machines. As shown in Table 2, the machine parameters are randomly generated: $\beta_{m} \in U(1,3), \quad \eta_{m} \in U(500,700), \quad t_{m}^{c} \in U(10,50)$. The flow line system with different 
configurations $\left(B_{m}=0,5,10\right)$ is simulated using the procedure shown in Fig. 2, and each procedure runs 500 times.

Table 2 Machine parameters

\begin{tabular}{|c|c|c|c|c|c|c|c|c|c|c|}
\hline$m$ & 1 & 2 & 3 & 4 & 5 & 6 & 7 & 8 & 9 & 10 \\
\hline$\eta_{m}$ & 618 & 684 & 627 & 548 & 540 & 644 & 587 & 641 & 516 & 552 \\
\hline$\beta_{m}$ & 2.3 & 1.5 & 2.9 & 1.3 & 2.9 & 1.5 & 1.1 & 2.5 & 1.9 & 1.1 \\
\hline$t_{m}^{c}$ & 40 & 21 & 35 & 16 & 23 & 22 & 18 & 40 & 50 & 17 \\
\hline
\end{tabular}

The simulation process was coded by $\mathrm{C} \#$ in a personal computer using a 64-bit Windows 10 operating system, a Dual-Core CPU, and an 8-GB RAM. As shown in Fig. 3, the average production time converges in tens of simulations. With confidence interval $\alpha=0.05$, all the trials have the same simulation error $\varepsilon<0.001$, which indicates the high accuracy of the simulation results.

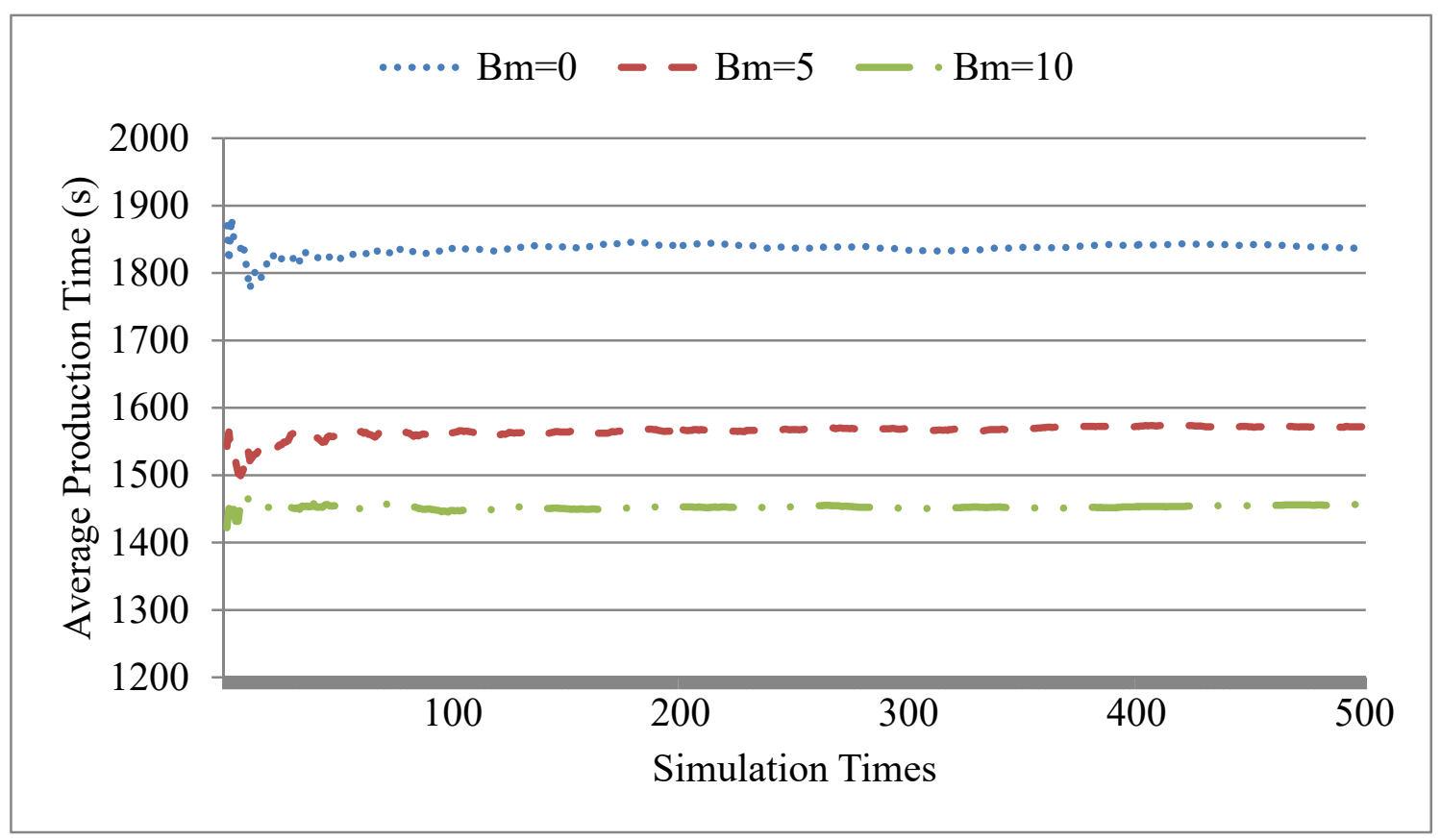

Fig. 3 Convergence of the production time

The production time after processing every product is recorded. Table $\mathbf{3}$ gives the regression results. It can be seen from the table that more than $99 \%$ of the original uncertainty has been explained by the polynomial. Moreover, the relative errors between the total production times are less than $2 \%$ (Table 4). Therefore, a second-order polynomial expression represents an excellent fit for approximating the simulation results.

Table 3 Regression results

\begin{tabular}{|c|c|c|c|}
\hline Configuration & $Q\left(x_{i t}\right)$ & $\begin{array}{c}\text { Adjusted } \\
\text { R-sq }\end{array}$ & RMSE \\
\hline$B_{m}=0$ & $8.35 \times 10^{-4} x^{2}+0.983 x+10$ & 0.98 & 6.63 \\
\hline$B_{m}=5$ & $5.27 \times 10^{-4} x^{2}+1.032 x+10$ & 0.99 & 4.23 \\
\hline$B_{m}=10$ & $3.67 \times 10^{-4} x^{2}+1.070 x+10$ & 0.99 & 3.04 \\
\hline
\end{tabular}


Table 4 Comparison of the total production time obtained from simulation and regression

\begin{tabular}{|c|c|c|c|}
\hline Configuration & $\begin{array}{c}\text { Simulation } \\
\text { result }\end{array}$ & $\begin{array}{c}\text { Regression } \\
\text { result }\end{array}$ & $\begin{array}{c}\text { Relative } \\
\text { error }\end{array}$ \\
\hline$B_{m}=0$ & 1837.44 & 1825.3 & $1.54 \%$ \\
\hline$B_{m}=5$ & 1571.87 & 1562.9 & $1.26 \%$ \\
\hline$B_{m}=10$ & 1456.15 & 1445.2 & $1.05 \%$ \\
\hline
\end{tabular}

Constraint (13) also requires the polynomial expression to fit well the product output in a period. In mass production, the output should be as large as possible without violating the capacity constraint. Assume that the output is larger than 200. Then, the deviation ratio between the simulation and the regression results can be calculated as follows:

$$
\text { ratio }=a b s\left[\frac{\left(\psi\left(n_{2}\right)-\psi\left(n_{1}\right)\right)-\left(Q\left(n_{2}\right)-Q\left(n_{1}\right)\right)}{\left(Q\left(n_{2}\right)-Q\left(n_{1}\right)\right)}\right] \times 100 \%, \quad n_{2}-n_{1}>200
$$

where $n_{1}$ and $n_{2}$ are the quantities of produced products.

To estimate the deviation ratio, 1,200 tuples of $n_{1}$ and $n_{2}$ are randomly generated using the Uniform $(1,1000)$ function. The majority of ratios are in the interval from $-3 \%$ to $3 \%$, and the percentage decreases when the system owns a larger buffer (Fig. 4). For the system with $B_{m}=0$, all ratios are within $\pm 3 \%$. This ensures the performance of this approximation approach in the integrated planning model.

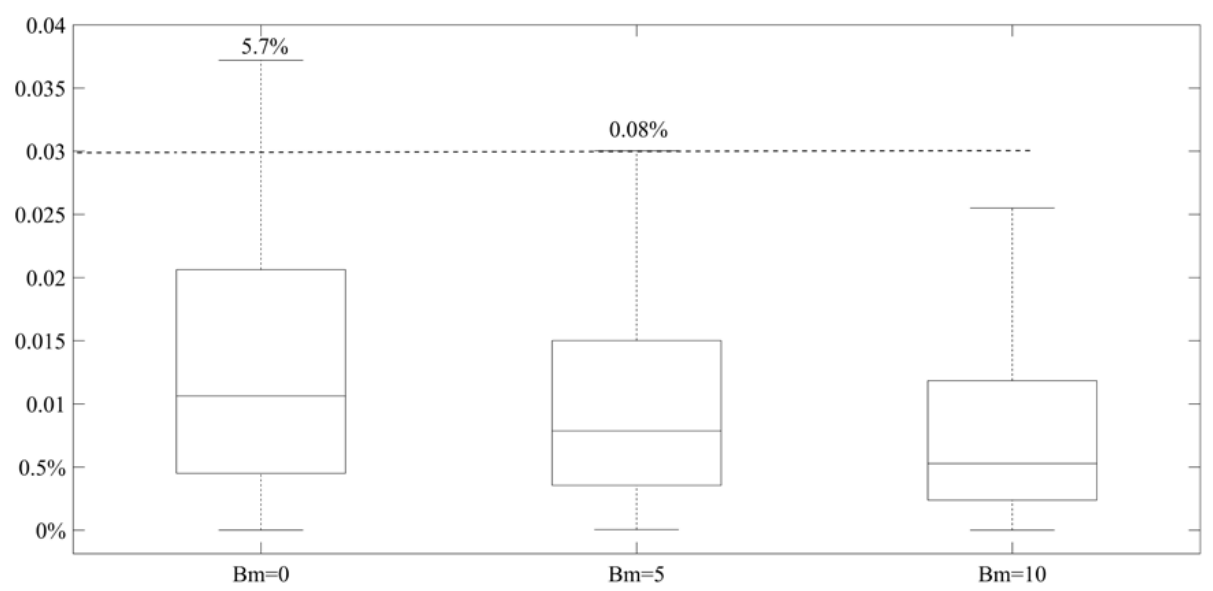

Fig. 4 Absolute values of deviation ratios

\section{Case study}

Re-consider the unreliable flow line described in Section 3 with the planning horizon being divided into 8 periods and each period having a maximum capacity of 400 time units. Two kinds of products are produced in the flow line and the demand for them are randomly generated between $(0,400)$ and are listed in Table 5. Some parameters (i.e. $\left.\beta_{m}, \eta_{m}, t_{m}^{c}\right)$ are listed in Table 2, and other can be seen in Table 6. Then, Lingo 11 is used to minimize the overall cost.

Table 5 Demand for products

\begin{tabular}{|c|c|c|c|c|c|c|c|c|}
\hline Period No. & 1 & 2 & 3 & 4 & 5 & 6 & 7 & 8 \\
\hline Product A & 103 & 62 & 162 & 5 & 17 & 385 & 295 & 89 \\
\hline Product B & 191 & 139 & 252 & 394 & 224 & 24 & 171 & 264 \\
\hline
\end{tabular}


Table 6 Other parameters of the flow line

\begin{tabular}{|c|c|c|c|c|c|c|c|}
\hline Parameters & $c_{i t}$ & $s_{i t}$ & $h_{i t}$ & $c_{s}^{p}$ & $t_{s}^{p}$ & $c_{m}^{c}$ & $t_{m}^{c}$ \\
\hline Values & 1 & 600 & 1 & 300 & 40 & 20 & 20 \\
\hline
\end{tabular}

\subsection{Comparison with the CLSP model}

Three levels (i.e. 0, 5, and 10) of the buffer capacity are considered in the CLSP-M models. Table 7 gives the capacity utilization and costs of the CLSP model and the CLSP-M models. The traditional CLSP model exhibits the lowest value of capacity utilization in all scenarios given in Table 7. With the maintenance activities and the multi-stage production characteristics considered in the integrated models, the capacity utilization reaches relatively high percentages, which are over $90 \%$. Some production activities are advanced or postponed in the CLSP-M models, thus the production cost is about 6-12\% greater when compared with the CLSP model.

In Table 7, the capacity utilization of the CLSP-M model decreases as the buffer capacity increases. It is because the flow line with a larger buffer capacity is always more production efficient and the demand for products is predetermined. Moreover, the more relaxed capacity constraint and higher flexibility of production and maintenance activities result from a lager buffer capacity. Therefore, it can be observed that the overall cost gradually decreases as the buffer capacity increases.

Table 7 Capacity utilization and costs of models

\begin{tabular}{|c|c|c|c|c|}
\hline Item/model & $\begin{array}{c}\text { CLSP } \\
\text { model }\end{array}$ & $\begin{array}{c}\text { CLSP-M model } \\
\left(B_{m}=0\right)\end{array}$ & $\begin{array}{c}\text { CLSP-M model } \\
\left(B_{m}=5\right)\end{array}$ & $\begin{array}{c}\text { CLSP-M model } \\
\left(B_{m}=10\right)\end{array}$ \\
\hline $\begin{array}{c}\text { Capacity } \\
\text { utilization }\end{array}$ & $69.43 \%$ & $96.42 \%$ & $92.56 \%$ & $91.34 \%$ \\
\hline Production cost & 11649 & 13063 & 12441 & 12351 \\
\hline Maintenance cost & 0 & 7412.3 & 7453.6 & 7477.2 \\
\hline Overall cost & 11649 & 20475.3 & 19894.6 & 19828.2 \\
\hline
\end{tabular}

\subsection{Validity of the capacity constraint}

Three sets of tests were executed, each set for another level of buffer capacity, and each test was simulated 500 times. Fig. 5 shows the simulation results of the violation of the capacity constraints in the periods. Violations are observed in the periods with tighter production constraints. The violation ratio does not exceed the level of the deviation ratios presented in Fig. 4. The violated capacity constraint phenomenon can be decreased by making slight adjustments to the existing decision results.

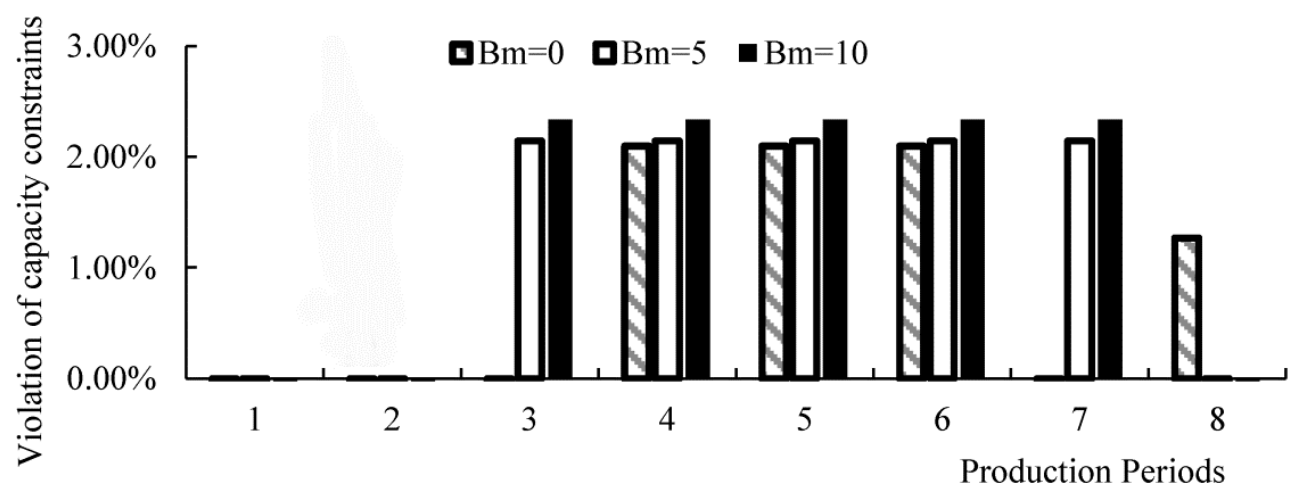

Fig. 5 Capacity constraint verification 


\section{Conclusion}

This paper extends the CLSP from a single-machine/single-stage system to an unreliable flow line. A mathematical model of the CLSP which considers maintenance operations is proposed with the aim of seeking a cost-optimal plan for both production and maintenance. In the model, synchronized preventive maintenance activities are performed to restore unreliable machines, and corrective maintenance is carried out on any machine in which an unplanned failure occurs. A regression-based approximation approach is proposed to obtain an expression of the production time, which is employed in the capacity constraints of the proposed model. Then, the model can be solved by any commercial optimization software. Experiments are conducted to reveal the effectiveness of the approximation approach. Lingo 11 is used to minimize the overall cost of the CLSP-M model. The results indicate that the capacity utilization is greatly improved compared with the CLSP model. Besides, the system configuration has non-negligible effects on the capacity utilization and cost, which should be considered in the integrated planning.

Future work would consider PM on the machine level, which would be more economic in cost. The main problem is to propose an approach to achieve such a production time of the flow line in which machines have different probability distribution. A reactive decisionmaking model of the CLSP-M considering rescheduling work tasks and PM is also an interesting research trend.

\section{Funding}

This investigation was supported by the National Natural Science Foundation of China (No. 51705263 and 51705262), Zhejiang Provincial Natural Science Foundation of China (No. LQ16G010002), and Ningbo Natural Science Foundation (No. 2017A610141). It was also sponsored by K. C. Wong Magna Fund of Ningbo University.

\section{REFERENCES}

[1] H. Wang, "A survey of maintenance policies of deteriorating systems," European Journal of Operational Research, vol. 139, no. 3, pp. 469-489, 2002. https://doi.org/10.1016/s0377 2217(01)00197-7

[2] A. T. de Almeida, R. J. P. Ferreira, and C. A. V. Cavalcante, "A review of the use of multicriteria and multi-objective models in maintenance and reliability," IMA Journal of Management Mathematics, vol. 26, no. 3, pp. 249-271, 2015. https://doi.org/10.1093/imaman/dpv010

[3] L. Weinstein, and C. H. Chung, "Integrating maintenance and production decisions in a hierarchical production planning environment," Computers \& Operations Research, vol. 26, no. 26, pp. 1059-1074, 1999. https://doi.org/10.1016/s0305-0548(99)00022-2

[4] X. Gu, S. Lee, X. Liang, M. Garcellano, M. Diederichs, and J. Ni, "Hidden maintenance opportunities in discrete and complex production lines," Expert Systems with Applications, vol. 40, no. 11, pp. 43534361, 9/1/, 2013. https://doi.org/10.1016/j.eswa.2013.01.016

[5] A. Arab, "Maintenance scheduling incorporating dynamics of production system and real-time information from workstations," Journal of Intelligent Manufacturing, vol. 24, no. 4, pp. 695-705, 2013. https://doi.org/10.1007/s10845-011-0616-3

[6] R. Ramezanian, M. Saidi-Mehrabad, and P. Fattahi, "MIP formulation and heuristics for multi-stage capacitated lot-sizing and scheduling problem with availability constraints," Journal of Manufacturing Systems, vol. 32, no. 2, pp. 392-401, 2013. https://doi.org/10.1016/j.jmsy.2013.01.002

[7] E. H. Aghezzaf, M. A. Jamali, and D. Ait-Kadi, “An integrated production and preventive maintenance planning model,” European Journal of Operational Research, vol. 181, no. 2, pp. 679-685, 2007. https://doi.org/10.1016/j.ejor.2006.06.032

[8] E. H. Aghezzaf, and N. M. Najid, Integrated production planning and preventive maintenance in deteriorating production systems: Elsevier Science Inc., 2008. https://doi.org/10.1016/j.ins.2008.05.007 
[9] M. Nourelfath, M. C. Fitouhi, and M. Machani, "An Integrated Model for Production and Preventive Maintenance Planning in Multi-State Systems,” IEEE Transactions on Reliability, vol. 59, no. 3, pp. 496506, 2010. https://doi.org/10.1109/tr.2010.2056412

[10] N. M. Najid, M. Alaoui-Selsouli, and A. Mohafid, "An integrated production and maintenance planning model with time windows and shortage cost," International journal of production research, vol. 49, no. 8, pp. 2265-2283, 2011. https://doi.org/10.1080/00207541003620386

[11] M. Nourelfath, and E. Châtelet, "Integrating production, inventory and maintenance planning for a parallel system with dependent components," Reliability Engineering \& System Safety, vol. 101, no. 1, pp. 59-66, 2012. https://doi.org/10.1016/j.ress.2012.02.001

[12] M.-C. Fitouhi, and M. Nourelfath, "Integrating noncyclical preventive maintenance scheduling and production planning for a single machine," International Journal of Production Economics, vol. 136, no. 2, pp. 344-351, 2012. https://doi.org/10.1016/j.ijpe.2011.12.021

[13] M. Alaoui-Selsouli, A. Mohafid, and N. Najid, "Lagrangian relaxation based heuristic for an integrated production and maintenance planning problem," International Journal of Production Research, vol. 50, no. 13, pp. 3630-3642, 2012. https://doi.org/10.1080/00207543.2012.671586

[14] M. Machani, and M. Nourelfath, "A variable neighbourhood search for integrated production and preventive maintenance planning in multi-state systems," International Journal of Production Research, vol. 50, no. 13, pp. 3643-3660, 2012. https://doi.org/10.1080/00207543.2012.671587

[15] Z. Lu, Y. Zhang, and X. Han, "Integrating run-based preventive maintenance into the capacitated lot sizing problem with reliability constraint," International Journal of Production Research, vol. 51, no. 5, pp. 1379-1391, 2013. https://doi.org/10.1080/00207543.2012.693637

[16] S. Wang, "Integrated model of production planning and imperfect preventive maintenance policy for single machine system," International Journal of Operational Research, vol. 18, no. 2, pp. 140-156, 2013. https://doi.org/10.1504/ijor.2013.056103

[17] A. Yalaoui, K. Chaabi, and F. Yalaoui, "Integrated production planning and preventive maintenance in deteriorating production systems," Information Sciences, vol. 278, pp. 841-861, 2014. https://doi.org/10.1016/j.ins.2014.03.097

[18] M. C. Fitouhi, and M. Nourelfath, "Integrating noncyclical preventive maintenance scheduling and production planning for multi-state systems," Reliability Engineering \& System Safety, vol. 121, no. 1, pp. 175-186, 2014. https://doi.org/10.1016/j.ress.2013.07.009

[19] S. Zhao, L. Wang, and Y. Zheng, "Integrating production planning and maintenance: an iterative method,” Industrial Management \& Data Systems, vol. 114, no. 2, pp. 162-182, 2014. https://doi.org/10.1108/imds-07-2013-0314

[20] H. B. Fakher, M. Nourelfath, and M. Gendreau, "Hybrid genetic algorithm to solve a joint production maintenance model," Ifac Papersonline, vol. 48, no. 3, pp. 747-754, 2015. https://doi.org/10.1016/j.ifacol.2015.06.172

[21] H. B. Fakher, M. Nourelfath, and M. Gendreau, "Joint production-maintenance planning in an imperfect system with quality degradation," in Industrial Engineering and Systems Management (IESM), 2015 International Conference on, 2015, pp. 910-919. https://doi.org/10.1109/iesm.2015.7380264

[22] E. H. Aghezzaf, P. L. Tam, and A. Khatab, "Optimizing Production and Imperfect Preventive Maintenance Planning's Integration in Failure-Prone Manufacturing Systems," Reliability Engineering [?] System Safety, vol. 145, no. 5, pp. 1-8, 2016. https://doi.org/10.1016/j.ress.2015.09.017

[23] H. Beheshti-Fakher, M. Nourelfath, and M. Gendreau, "Joint planning of production and maintenance in a single machine deteriorating system," IFAC PapersOnLine, vol. 49, no. 12, pp. 745-750, 2016. https://doi.org/10.1016/j.ifacol.2016.07.863

[24] F. Hnaien, F. Yalaoui, A. Mhadhbi, and M. Nourelfath, "A mixed-integer programming model for integrated production and maintenance," IFAC-PapersOnLine, vol. 49, no. 12, pp. 556-561, 2016. https://doi.org/10.1016/j.ifacol.2016.07.694

[25] M. Erfanian, and M. Pirayesh, "Integration aggregate production planning and maintenance using mixed integer linear programming," in Industrial Engineering and Engineering Management (IEEM), 2016 IEEE International Conference on, 2016, pp. 927-930. https://doi.org/10.1109/ieem.2016.7798013

[26] M. Nourelfath, N. Nahas, and M. Ben-Daya, "Integrated preventive maintenance and production decisions for imperfect processes," Reliability Engineering \& System Safety, vol. 148, pp. 21-31, 2016. https://doi.org/10.1016/j.ress.2015.11.015 
[27] F. Shamsaei, and M. V. Vyve, "Solving integrated production and condition-based maintenance planning problems by MIP modeling," Flexible Services \& Manufacturing Journal, vol. 29, no. 2, pp. 1-19, 2016. https://doi.org/10.1007/s10696-016-9244-8

[28] H. Beheshti Fakher, M. Nourelfath, and M. Gendreau, "A cost minimisation model for joint production and maintenance planning under quality constraints," International Journal of Production Research, vol. 55, no. 8, pp. 2163-2176, 2017. https://doi.org/10.1080/00207543.2016.1201605

[29] W. Abdul-Kader, "Capacity improvement of an unreliable production line_ - an analytical approach," Computers \& Operations Research, vol. 33, no. 6, pp. 1695-1712, 2006. https://doi.org/10.1016/j.cor.2004.11.015

[30] J. E. Diaz, and J. Handl, "Implicit and explicit averaging strategies for simulation-based optimization of a real-world production planning problem," Informatica, vol. 39, no. 2, pp. 161, 2015.

[31] A. Caggiano, G. Bruno, and R. Teti, "Integrating Optimisation and Simulation to Solve Manufacturing Scheduling Problems," Procedia Cirp, vol. 28, pp. 131-136, 2015. https://doi.org/10.1016/j.procir.2015.04.022

[32] Y. Chu, F. You, J. M. Wassick, and A. Agarwal, "Integrated planning and scheduling under production uncertainties: Bi-level model formulation and hybrid solution method," Computers \& Chemical Engineering, vol. 72, pp. 255-272, 2015. https://doi.org/10.1016/j.compchemeng.2014.02.023

[33] T. Ponsignon, and L. Mönch, "Simulation-based performance assessment of master planning approaches in semiconductor manufacturing," Omega, vol. 46, pp. 21-35, 2014. https://doi.org/10.1016/j.omega.2014.01.005

[34] S. Mehrjoo, and M. Bashiri, "An application of principal component analysis and logistic regression to facilitate production scheduling decision support system: an automotive industry case," Journal of Industrial Engineering International, vol. 9, no. 1, pp. 14, 2013. https://doi.org/10.1186/2251-712x-9-14

[35] N. Stricker, A. Pfeiffer, E. Moser, B. Kádár, G. Lanza, and L. Monostori, "Supporting multi-level and robust production planning and execution,” CIRP Annals - Manufacturing Technology, vol. 64, no. 1, pp. 415-418, 2015. https://doi.org/10.1016/j.cirp.2015.04.115

[36] D. V. Gyulai, A. S. Pfeiffer, and L. S. Monostori, "Robust production planning and control for multistage systems with flexible final assembly lines," International Journal of Production Research, vol. 55, no. 13, pp. 3657-3673, 2017. https://doi.org/10.1080/00207543.2016.1198506

[37] K. Wu, "New results in factory physics-insights from the underlying structures of manufacturing systems," Georgia Institute of Technology, 2009.

Submitted: $\quad 21.5 .2019$

Accepted: $\quad 27.11 .2019$
Wenwen Lin

1. Faculty of Mechanical Engineering \& Mechanics, Ningbo University, Ningbo Zhejiang 315211, China

2. Part Rolling Key Laboratory of Zhejiang Province, Ningbo University, Ningbo Zhejiang 315211, China

308 Xiushan Engineering Hall, 818

Fenghua Road, Ningbo, Zhejiang, 315211, China

Yuejun Zhang

Faculty of Mechanical Engineering \& Mechanics, Ningbo University, Ningbo Zhejiang 315211, China 